\title{
Black holes, dark matter spikes, and constraints on simplified models with $\boldsymbol{t}$-channel mediators
}

\author{
Pearl Sandick ${ }^{*}$ and Takahiro Yamamoto ${ }^{\dagger}$ \\ Department of Physics and Astronomy, University of Utah, Salt Lake City, Utah 84112, USA \\ Kuver Sinha \\ Department of Physics and Astronomy, University of Oklahoma, Norman, Oklahoma 73019, USA
}

(Received 18 September 2017; published 2 August 2018)

\begin{abstract}
A possible density spike of dark matter (DM) in the subparsec region near the supermassive black hole at the Galactic center could have a significant effect on indirect signals of dark matter annihilation. Here we explore the impacts of the modeling of the dark matter distribution in the inner Galaxy on gamma-ray signals of dark matter annihilation. We consider a range of values for relevant astrophysical parameters that describe the dark matter profile, including a possible spike, and quantify the dependence of the gamma-ray flux on these choices. We consider both an idealized case where no depletion of the spike has occurred since its formation, as well as a case where the spike is significantly depleted over time due, e.g., to gravitational interactions with baryons. We also consider a range of power-law descriptions of the dark matter profile outside of the spike region. Taking Fermi-LAT data for the gamma-ray flux from the point source 3FGL J1745.6-2859c (Sgr A*), we calculate the resulting constraints on generic models of DM, allowing for the possibility of a non-negligible velocity-dependent component of the annihilation cross section. We find that for very depleted spikes, the spike itself would contribute insignificantly to a dark matter annihilation signal, though constraints on dark matter models are still possible depending on the power law behavior of the dark matter profile on larger scales. For idealized spikes, we quantify the influence of the spike, as well as the larger-scale dark matter profile, on the gamma-ray flux from dark matter annihilations and on the constraints on the properties of particle dark matter from observations of the Galactic center point source.
\end{abstract}

DOI: 10.1103/PhysRevD.98.035004

\section{INTRODUCTION}

The particle nature of dark matter (DM) is an area of intense investigation which has the potential to shed light on fundamental questions about the standard model (SM), especially the hierarchy problem. For DM candidates with weak-scale couplings and mass, a calculation of the relic density automatically yields a value that is close to the measured dark matter abundance. This striking fact, a success of the so-called weakly interacting massive particle (WIMP) paradigm, reinforces the possibility that DM is deeply connected to new physics at the weak scale. The indirect detection of the products of DM annihilation or

\footnotetext{
*sandick@physics.utah.edu

t.yamamoto.1777@gmail.com

"kuver.sinha@ou.edu
}

Published by the American Physical Society under the terms of the Creative Commons Attribution 4.0 International license. Further distribution of this work must maintain attribution to the author(s) and the published article's title, journal citation, and DOI. Funded by SCOAP . decay are one potentially fruitful way to investigate the properties of DM. Indeed, if DM annihilations occurred in the early Universe, it is possible that we could observe the products of annihilations occurring today.

Indirect detection of WIMPs in the Milky Way halo has been a major endeavor over many years. The gamma-ray flux $\Phi$ coming from WIMP annihilation is proportional to the line-of-sight integral of the square of the DM density,

$$
\Phi \sim \int \rho^{2}(r) d r
$$

Since the Galactic center is expected to have a very high density of DM, it has been a much-studied source for indirect detection of DM.

The formation of black holes at the centers of DM halos, and in particular the supermassive black hole at the center of our Galaxy [1,2], can significantly modify the DM profile and affect the observed gamma-ray flux from that region. Gondolo and Silk showed [3] that if the black hole grows adiabatically at the center of a cusp with a power-law profile, 


$$
\rho(r) \sim r^{-\gamma_{c}} \quad(\text { cusp profile })
$$

a DM spike can form close to the black hole, with a density profile given by

$$
\rho(r) \sim r^{-\gamma_{\mathrm{sp}}} \quad(\text { spike profile })
$$

with $\gamma_{\mathrm{sp}}>\gamma_{c}$. Such a spike causes an increase in $\Phi$ due to the enhanced density $\rho$ in Eq. (3) at small radii. In fact, as $r \rightarrow 0$ the DM density profile diverges, but the divergence is cut off by the black hole horizon and smoothed near it due to the effects of DM annihilation.

The account above is an idealized case, since a DM spike could be destroyed or smoothed by various effects [4-8]. In galactic nuclei, stars have much larger kinetic energy than DM particles, and interactions between them cause DM to be heated up. The gravitational interaction between stars near the black hole and the DM spike can thus cause damping, which affects the spike parameters, including the power-law behavior and the spike radius. The astrophysical parameters that describe the DM spike are a topic of ongoing debate, with a fairly broad range of plausible possibilities.

The purpose of this paper is to investigate contributions of annihilations in a DM spike, as well as outside the spike itself, to the gamma-ray flux from the Galactic center point source. Specifically, we investigate different spike profiles (i.e., spike formation histories) to determine whether the expectation of an enhanced signal due to the presence of a spike is robust. We consider cases where the DM profile is an idealized spike, which has not changed significantly since its formation, as well as cases where the spike has been depleted over time, due to gravitational interactions with baryons. Since the dark matter profile is, in general, not well constrained, we consider a range of power-law descriptions of the dark matter profile outside of the spike region, as well. In the following study, we quantify the influence of the dark matter profile on the gamma-ray flux from dark matter annihilations and on the constraints on the properties of particle dark matter from observations of the Galactic center point source.

As has been discussed in $[9,10]$, in the region very near the Galactic center, the gravitational influence of the black hole affects not only the DM profile, but also the DM velocity dispersion. Even DM models in which the annihilation cross section today is completely velocitysuppressed may lead to non-negligible gamma-ray signals from the Galactic center, where the velocities can be quite large. Here, we investigate a range of DM models with both velocity-independent as well as velocity-dependent contributions to the annihilation cross section. Finally, we present a concrete example of a model in which the conclusions from gamma-ray data depend strongly on the details of the DM spike: a simplified model of fermionic
DM coupled to standard model fermions via charged scalars $[11,12]$.

Indirect detection of DM from a spike near the central black hole of our galaxy has been studied by several authors in different contexts in particle physics. Recently, $[9,13,14]$ have studied these issues in the context of the Galactic center excess and for DM models with $p$-wave annihilation for an idealized spike. Indirect detection of DM with a velocity-dependent annihilation cross section has been studied by [10], in models of nonthermal DM by [15], and in the context of dark stars by [16-18]. Meanwhile, spikes at the centers of dwarf galaxies have recently been constrained by [19,20], and in M87 by [21].

Here, we expand on the studies of DM annihilation near the Milky Way Galactic center. We find that the size of the spike, denoted by the spike radius, $r_{\mathrm{sp}}$, and the steepness of the profile both inside the spike, parametrized by $\gamma_{\mathrm{sp}}$, and outside (in the cusp), parametrized by $\gamma_{c}$, have a strong effect on the resulting constraints on DM models.

Our work suggests that a more careful study of the astrophysics of DM spikes near black holes, specifically in the neighborhood of the supermassive black hole (SMBH) at the center of our Galaxy, is warranted. The wide range of plausible spike parameters results in significant variation in the space of DM constraints.

The paper is structured as follows. In Sec. II, we discuss the parameters that describe the DM spike near the black hole. In Sec. III, we discuss our main results in general DM models. In Sec. IV, we describe our results in the context of a simplified DM model, in which dark matter annihilates via $t$-channel exchange of charged mediators. In Sec. V, we briefly discuss what can be learned about the DM spike under the assumption of a particular DM model, in this case one designed to explain the excess of $\mathrm{GeV}$ photons from the Galactic center [22-24]. We end with our Conclusions.

\section{DARK MATTER SPIKE NEAR THE SUPERMASSIVE BLACK HOLE}

In this section, we discuss the profile of a DM spike near the SMBH in the inner subparsec region of our Galaxy. This type of DM spike has been studied by many groups, beginning with the work of Gondolo and Silk [3]. Unless otherwise noted, we remain agnostic about the nature of DM, and parametrize its annihilation cross section as [25]

$$
\langle\sigma v\rangle \approx c_{0}+c_{1}\left(\frac{v}{c}\right)^{2}
$$

where $c_{0}$ is the velocity-independent $s$-wave contribution, and $c_{1}$ is the $v^{2}$-suppressed contribution. We note that the velocity-suppressed terms arise from both $s$-wave and $p$-wave matrix elements.

We consider a SMBH at the center of our Galaxy [1] with mass, $M_{b h}$, and Schwarzchild radius, $r_{\text {Sch. }}$, 


$$
\begin{aligned}
M_{b h} & =4 \times 10^{6} M_{\odot} \\
r_{\text {Sch }} & =4 \times 10^{-7} \mathrm{pc} .
\end{aligned}
$$

If the growth of the SMBH was adiabatic, and assuming collisionless dark matter particles, one finds that an original DM cusp with density profile $\rho(r) \propto r^{-\gamma_{c}}$ becomes contracted into a spike with profile $\rho(r) \propto r^{-\gamma_{\mathrm{sp}}}$, with $\gamma_{\mathrm{sp}}>\gamma_{c}$, at small radii $[3,4]$. In fact, at the smallest radii, just outside $r_{\text {Sch. }}$, the DM density likely attains a maximum or plateau value. There are thus three distinct regions of the DM density profile, which will be discussed in detail in Sec. II B. Specifically, the profile is given by the analytic form

$$
\rho(r)= \begin{cases}\rho\left(r_{\text {core }}\right) & 10 r_{\mathrm{Sch}}<r \leq r_{\text {core }}(\mathrm{III}) \\ \rho_{0}\left(r / r_{\mathrm{sp}}\right)^{-\gamma_{\mathrm{sp}}} & r_{\mathrm{core}}<r \leq r_{\mathrm{sp}}(\mathrm{II}) \\ \rho_{0}\left(r / r_{\mathrm{sp}}\right)^{-\gamma_{c}} & r_{\mathrm{sp}}<r(\mathrm{I})\end{cases}
$$

Here, $r_{\mathrm{sp}}$ and $r_{\text {core }}$ denote the spike and core radii, respectively. The profile depends on the steepness parameters $\gamma_{\mathrm{sp}}$ and $\gamma_{c}$.

The formation of a DM spike is contingent on several conditions, detailed e.g., in $[3,4]$. After its formation, the DM spike may be depleted due to gravitational interactions with stars near the Galactic center, or disrupted due to halo mergers, either of which can substantially reduce the steepness of the spike [4]. Here we consider the case of an idealized (undepleted) spike, as well as a spike that has been depleted due to gravitational interactions with stars. For the latter, we follow the parametrization of [8].

We first give details about the spike and core radii, $r_{\mathrm{sp}}$ and $r_{\text {core }}$, then we describe the physics of the profile for each of the three regions.



\section{A. Spike radius $\left(r_{\mathrm{sp}}\right)$ and depletion effects}

In the idealized case, the spike radius does not evolve in time and is given by

$$
r_{\mathrm{sp}}(t)=r_{\mathrm{sp}}(0) \sim 0.2 r_{h} \quad(\text { Idealized }) .
$$

Here, $r_{h}$ denotes the radius of gravitational influence of the black hole,

$$
r_{h} \equiv \frac{G M_{b h}}{\sigma^{2}},
$$

where $G$ is Newton's constant and $\sigma$ is the one-dimensional velocity dispersion. $M_{b h}$ is related to $\sigma$ through the empirical relation [26]

$$
\frac{M_{b h}}{10^{8} M_{\odot}}=(1.66 \pm 0.24)\left(\frac{\sigma}{200 \mathrm{~km} \mathrm{~s}^{-1}}\right)^{4.86 \pm 0.43} .
$$

For the central values in Eq. (9), we obtain

$$
\begin{aligned}
\sigma & =93 \mathrm{~km} / \mathrm{s} \\
r_{h} & =1.99 \mathrm{pc}
\end{aligned}
$$

for the DM velocity dispersion and the radius of influence of the black hole. In the idealized case, this leads to a spike radius of

$$
r_{\mathrm{sp}}=0.40 \mathrm{pc} \quad(\text { Idealized }),
$$

denoted as $r_{\mathrm{sp}}(0)$ in Fig. 1.

Gravitational interactions between DM and baryons will lead to changes from the idealized case of Eq. (7). Stars in the galactic nucleus have much larger kinetic energies than the DM particles, and the interactions between the two tend

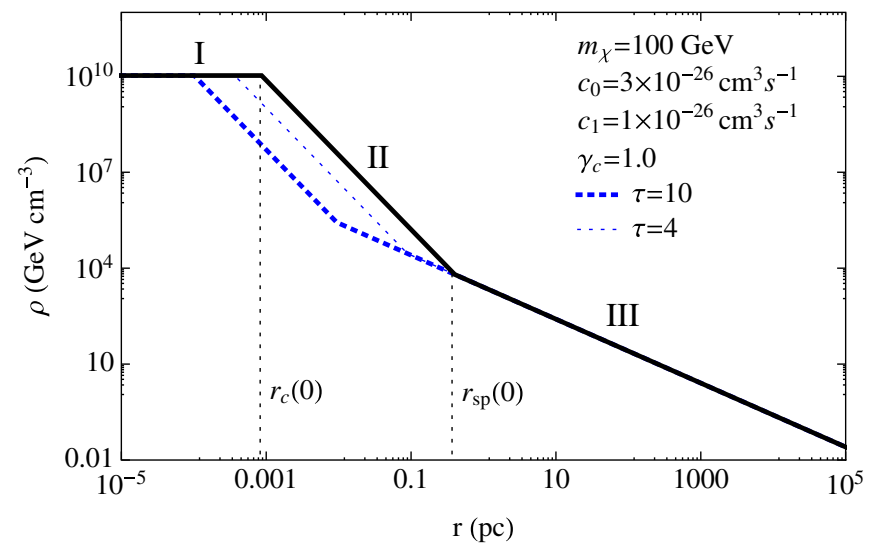

FIG. 1. Example dark matter profiles for $\gamma_{c}=1.0$, and assuming adiabatic contraction such that $\gamma_{\mathrm{sp}}$ is calculated according to Eq. (18), for a benchmark case with thermal, primarily $s$-wave annihilating dark matter. In the left panel, we show the spike radius $\left(r_{\mathrm{sp}}\right)$ for the relevant range of $\gamma_{c}$, while in the right panel, we present the density profiles. In each panel, we show the idealized case (black solid), a depleted case with $\tau=10$ (blue dashed) as considered throughout the rest of this study, and, for reference, a depleted case with $\tau=4$ (blue dotted), where $\tau$ denotes the time since the spike formed in units of the heating time (details in text). 
to heat up the DM. This leads to a dampening of the spike [5]. The decay of the spike can be described roughly as

$$
\rho(r, t) \approx \rho(r, 0) e^{-\tau / 2},
$$

where $\tau$ is the time since the spike formed in units of the heating time, $t_{\text {heat }}[8]$. Throughout this study, we assume $t_{\text {heat }}=10^{9}$ years, implying $\tau=10$ [27], though we note that these values are not well constrained.

The evolution of the spike radius can be described as [8]

$r_{\mathrm{sp}}(t)=r_{\mathrm{sp}}(0) \times \exp \left(\frac{-\tau}{2\left(\gamma_{\mathrm{sp}}-\gamma_{c}\right)}\right) \quad($ Depleted $)$,

where $r_{\mathrm{sp}}(0)=0.2 r_{h}$ is the initial value of the spike radius. Typical values for the depleted spike radius today $\left(t_{0}\right)$ are

$r_{\mathrm{sp}}\left(t_{0}\right)=0.0094 \mathrm{pc}$ for $\gamma_{c}=1.0$

$r_{\mathrm{sp}}\left(t_{0}\right)=0.0015 \mathrm{pc}$ for $\gamma_{c}=1.5 \quad$ (Depleted),

assuming $\gamma_{\mathrm{sp}}$ arises solely due to adiabatic growth of the black hole, as described in Sec. II B. Note that the spike radius in the depleted case is much smaller than in the idealized case, and also that there is significant variation in the spike radius depending on $\gamma_{c}$.

In Fig. 1 we fix $\gamma_{c}=1.0$ and present an idealized spike (solid black), a depleted spike with $\tau=10$ (blue dashed), and a depleted spike with $\tau=4$, i.e., $t_{\text {heat }}=2.5 \times 10^{9}$ years, (blue dotted). In all cases, we take $m_{\chi}=100 \mathrm{GeV}$, and consider a benchmark case with thermal, primarily $s$-wave annihilating DM. In the left panel, we show the spike radius, $r_{\mathrm{sp}}$, as a function of $\gamma_{c}$. The spike radius can vary over more than two orders of magnitude, depending on whether the spike is idealized or depleted. In the case of a depleted spike with $\tau=10$, there can even be nearly an order of magnitude variation of the spike radius, depending on the form of the cusp from which the spike was formed. Profiles with large $\gamma_{c}$ end up with smaller spikes than profiles with smaller $\gamma_{c}$. In the right panel, we show the DM density as a function of radius, highlighting regions I, II, and III from Eq. (6). Vertical dotted lines indicate the core and spike radii for an idealized spike. We note that the idealized spike profile agrees well with other profiles in the literature, e.g., $[9,13,14,28]$, while our depleted spike profiles follow expectations from $[5,6,8,10,27]$. From the right panel, it is evident that even for $\tau=4$ (blue dotted), the spike is significantly depleted relative to the idealized case, and for $\tau=10$ (blue dashed), the depletion is considerably more severe. Spikes of such varying size lead to a large range of predicted indirect signals of DM annihilation.

Throughout the rest of this study, we restrict our attention on the case of $\tau=10$ to demonstrate the effects of depletion. Unless otherwise noted, depleted spikes considered here have spike radii described by Eq. (13), with $\gamma_{\mathrm{sp}}$ according to the adiabatic expectation, as discussed below.

\section{B. Dark matter density profile}

In this section, we discuss in detail the halo profile of the DM spike, starting from the outermost region and going to the innermost region.

Region I $\left(r>r_{\mathrm{sp}}\right)$ : Typical DM halo profiles, such as Navarro Frenk White (NFW) [29], are characterized by a double power law profile. For the Milky Way, at radii less than $\sim 10 \mathrm{kpc}$, the profile is a single power law, which we take to be relevant for $r>r_{\mathrm{sp}}$, the radius inside which the spike is significant. We may therefore parametrize the DM profile in Region I as

$$
\rho(r)=\rho_{0}\left(\frac{r}{r_{\mathrm{sp}}}\right)^{-\gamma_{c}} \text { for } r_{\mathrm{sp}}<r .
$$

The normalization of the density profile $\rho_{0}$ is set by extrapolating inwards from the solar radius

$$
\rho_{0}=\rho_{\odot}\left(\frac{r_{\odot}}{r_{\mathrm{sp}}}\right)^{\gamma_{c}}
$$

where we take the density at the solar radius to be $\rho_{\odot}=0.3 \mathrm{GeV} / \mathrm{cm}^{3}$.

N-body simulations that include only DM (and no baryonic matter) generally favor inner slopes of $\gamma_{c} \approx 1$, which is the canonical NFW value. However, baryonic interactions affect the profile in the inner $10 \mathrm{kpc}$ of our galaxy, and can substantially steepen the power-law behavior [30-34]. Furthermore, observations are compatible with $\gamma_{c}$ as large as at least 1.5 [34]. Here we consider a range of cusp exponents, allowing values of $\gamma_{c} \in[1.0,1.5]$.

Region II $\left(r_{\text {core }}<r<r_{\mathrm{sp}}\right)$ : The spike profile itself is also parametrized as a simple power law,

$$
\rho(r)=\rho_{0}\left(\frac{r}{r_{\mathrm{sp}}}\right)^{-\gamma_{\mathrm{sp}}} \quad \text { for } r_{\text {core }}<r \leq r_{\mathrm{sp}},
$$

where the spike slope, $\gamma_{\mathrm{sp}}$ may or may not be directly related to the cusp slope, $\gamma_{c}$. For collisionless DM forming a spike due to the adiabatic growth of the black hole, the spike slope obeys the relation

$$
\gamma_{\mathrm{sp}}=\frac{9-2 \gamma_{c}}{4-\gamma_{c}},
$$

which yields a value $\gamma_{\mathrm{sp}} \approx 2.3-2.4$ for $1.0 \leq \gamma_{c} \leq 1.5$. This relation holds for a central black hole that grows adiabatically from a small seed.

However, the spike slope may be significantly different than the adiabatic expectation under different assumptions. 
If the black hole appeared instantaneously, then one obtains $\gamma_{\mathrm{sp}}=4 / 3$ [4]. Black hole mergers at the center of the progenitor halo can give $\gamma_{\mathrm{sp}}=1 / 2$, a value that is also obtained if the black hole grows away from the center of the DM distribution [4]. As above, we focus on the effect of stellar heating, which could result in a final equilibrium value as low as $\gamma_{\mathrm{sp}} \approx 1.5[5,7]$. Note that more recent work by the author of [5], namely [8], indicates that the effect of stellar heating will be a decrease in $r_{\mathrm{sp}}$, rather than a direct decrease in $\gamma_{\mathrm{sp}}$ with $r_{\mathrm{sp}}$ unchanged. In the remainder of the paper, we primarily follow [8], though we briefly comment on the possibility of a reduced value of $\gamma_{\mathrm{sp}}$ relative to the adiabatic expectation, as was explored, e.g., in [13].

Region III $\left(r<r_{\text {core }}\right)$ : At very small radii, the DM density can reach very high values. However, that implies large values of the annihilation cross section, which acts to reduce the density. We make the conservative assumption that an annihilation plateau is formed in this region, with

$$
\rho(r)=\rho\left(r_{\text {core }}\right) \text { for } 10 r_{\text {Sch }}<r \leq r_{\text {core }},
$$

where the relevant inner radius is related to the Schwarzschild radius of the black hole, and the outer radius is the core radius, defined by the relation

$$
\frac{\rho\left(r_{\text {core }}\right)}{m_{\chi}}\langle\sigma v\rangle \approx\left(\tau t_{\text {heat }}\right)^{-1},
$$

which depends on the thermally averaged annihilation cross section times velocity, $\langle\sigma v\rangle$, and the WIMP mass, $m_{\chi}$.

We note that in the general case of arbitrary velocity anisotropy, instead of circular particle orbits, a cusp with $\rho \propto r^{(-\beta-1 / 2)}$ may develop in the center, where $\beta$ is the anisotropy coefficient [35]. Though the cusp is expected to be very weak, it may further enhance the flux of DM annihilation products from the very central region of the Galaxy [14]. Here we take the simple limit of circular orbits, in which case there is a flat plateau as depicted in Fig. 1.

Finally, we assume a virialized halo such that

$$
\left(\frac{v}{c}\right)^{2}=\frac{r_{\mathrm{Sch}}}{2 r}
$$

Since the ratio $v / c$ appears in the partial wave expansion of the annihilation cross section, given by Eq. (4), the annihilation cross section is therefore position-dependent, and the velocity-suppressed contribution can become large near the SMBH. In fact, from Eq. (20), we see that $r_{\text {core }}$, and therefore also $\rho(r)$ for $r<r_{\text {core }}$, may be sensitive to the velocity-suppressed contribution to the annihilation cross section, and will in general vary with the coefficient $c_{1}$ even for fixed $c_{0}$.

\section{RESULTS: GENERIC DM MODEL}

In this section, we present our results for the sensitivity of constraints on the properties of dark matter to assumptions about the DM density profile in the inner galaxy.

We begin by discussing our calculation of the gammaray flux from dark matter annihilations in the Galactic center region. The differential flux of gamma rays from a given angular direction $d \Omega$ produced by the annihilation of Majorana DM, $\chi$, is given by

$$
\begin{aligned}
\frac{d \Phi_{\gamma}}{d \Omega d E}= & \frac{1}{2} \frac{r_{\odot}}{4 \pi}\left(\frac{\rho_{\odot}}{m_{\chi}}\right)^{2} \int_{\text {l.o.s. }} \frac{d s}{r_{\odot}}\left(\frac{\rho(r(s, \theta))}{\rho_{\odot}}\right)^{2} \\
& \times \sum_{f}\langle\sigma v\rangle_{f} \frac{d N_{\gamma}^{f}}{d E} .
\end{aligned}
$$

Here, $r(s, \theta)=\left(r_{\odot}^{2}+s^{2}-2 r_{\odot} s \cos \theta\right)^{1 / 2}$ is the radial Galactic coordinate, and $\theta$ is the angle between the direction of the line of sight, $s$, and the axis connecting the Earth and the Galactic center. $d N_{\gamma}^{f} / d E$ is the spectrum of photons coming from annihilation to a final state $f$, and is computed with PyTHIA [36]. We note that the usual separation between the calculation of the astrophysical $J$-factor and the annihilation cross section is no longer applicable here, since the annihilation cross section itself depends on position, as discussed above.

If the DM spike is a bright and compact enough source of photons, it could appear as a point source to the Fermi$L A T$. We consider the integrated flux from 1 to $100 \mathrm{GeV}$ for the Fermi 3FGL source J1745.6-2859c (Sgr A*), which we denote as $\Phi_{\text {Fermi }}=2.18 \times 10^{-8}$ photons $/ \mathrm{cm}^{2} \mathrm{~s}$. In fact, the bulk of the contribution to the gamma-ray flux from Sgr $\mathrm{A}^{*}$ is from standard astrophysics, rather than DM annihilation. But even in this case, any photons from DM annihilation in that region of the sky would necessarily contribute to the observed point source spectrum (see, e.g., [37]). In this spirit, we consider a model to be excluded only if the flux from the spike alone exceeds $\Phi_{\mathrm{Fermi}}$.

That said, given the very large range of expectations for the form of the dark matter profile, including a possible spike, we are primarily interested here in order of magnitude estimates, and prefer to remain agnostic about the nature of the DM. Rather than assuming a specific final state to which dark matter annihilates, we choose as a benchmark value for the integrated photon count $N=1$, with the flux scaling $\propto N$, and integrate over a fixed angular acceptance of $0.1^{\circ}$ [38]. Note that for common final states $b \bar{b}, \tau^{+} \tau^{-}$, and $\mu^{+} \mu^{-}, N \approx 13,2.5$, and 0.5 , respectively.

In Fig. 2 we present the point source flux, $\Phi$, from DM annihilation in units of $\Phi_{\mathrm{Fermi}}$ in the $\left(c_{0}, c_{1}\right)$ plane for $m_{\chi}=$ $100 \mathrm{GeV}$ for the case of a depleted spike. In the left panel, $\gamma_{c}=1.0$, resulting in a flux that is $\gtrsim 3$ orders of magnitude smaller than the observational sensitivity. In the right panel, $\gamma_{c}=1.5$, in which case the observed gamma-ray flux 

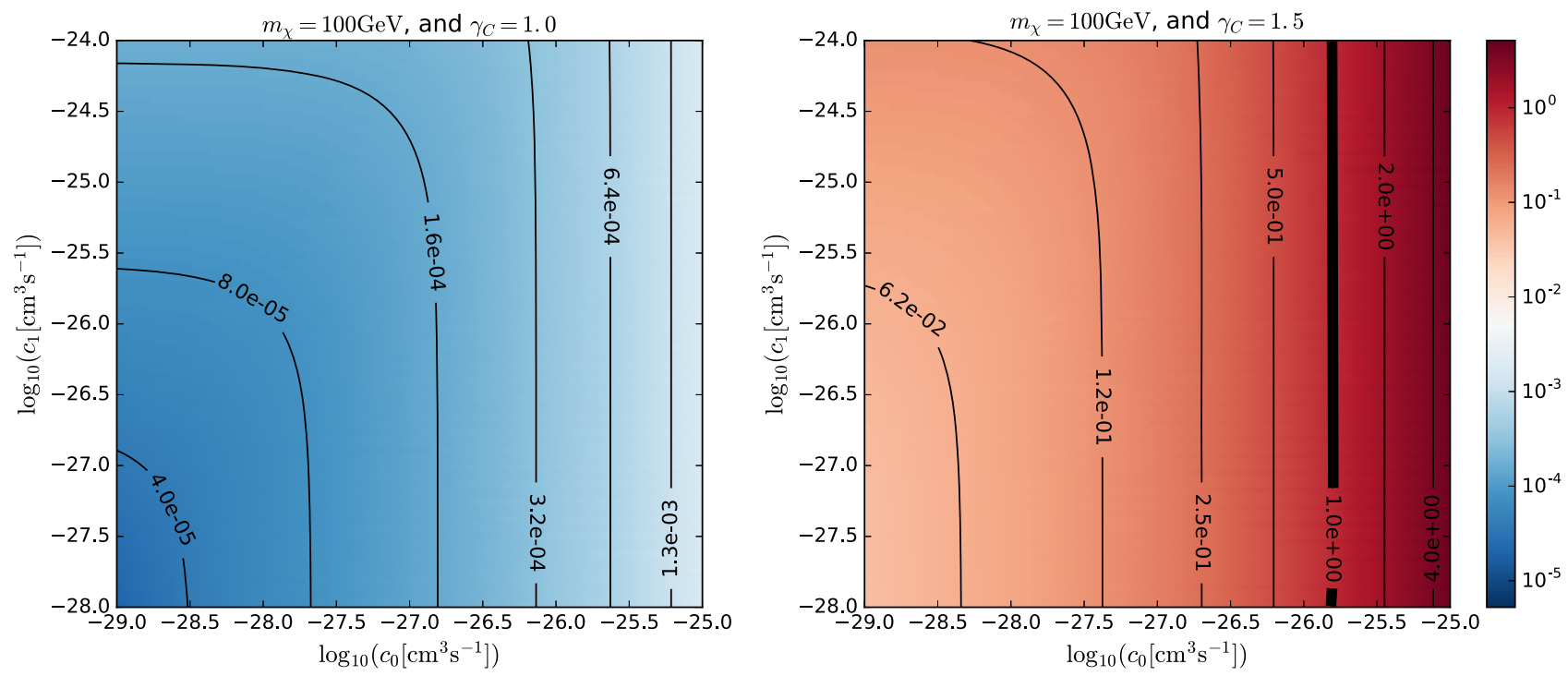

FIG. 2. Depleted Spike, $100 \mathrm{GeV}$ DM: Contours of the integrated flux $\Phi$ in units of $\Phi_{\text {Fermi }}=2.18 \times 10^{-8} \mathrm{photons} / \mathrm{cm}^{2} \mathrm{~s} \mathrm{coming}$ from the source 3FGL J1745.6-2859c (Sgr A*), in the energy range 1-100 GeV, and assuming an integrated photon count $N=1$. The dark matter mass is $100 \mathrm{GeV}$, and the annihilation cross section is parametrized by Eq. (4). The spike profile is given by Eq. (6). The spike radius is given by the depleted case in Eq. (13). The spike power law outside the spike radius is given by $\gamma_{c}=1.0$ (left panel) and $\gamma_{c}=1.5$ (right panel). The bold line in the right panel shows the contour $\Phi=\Phi_{\text {Fermi }}$.

constrains models with annihilation cross section $c_{0} \gtrsim$ $10^{-26} \mathrm{~cm}^{3} \mathrm{~s}^{-1}$ for $N=1$, and could reach to much lower annihilation cross sections for larger $N$. For $\gamma_{c}<1.5$, the predicted flux from DM annihilation is obviously smaller: For example, for $\gamma_{c}=1.3, \Phi \ll \Phi_{\mathrm{Fermi}}$ for the entirety of the $\left(c_{0}, c_{1}\right)$ parameter space explored for $N=1$, though $\Phi \approx 0.1 \times \Phi_{\text {Fermi }}$ for $c_{0} \approx 10^{-25} \mathrm{~cm}^{3} \mathrm{~s}^{-1}$, so for a final state with a large integrated photon count in the relevant energy range, such as $b \bar{b}$, some relevant DM constraints are possible for $\gamma_{c} \approx 1.3$ and $m_{\chi}=100 \mathrm{GeV}$.

Another important result from Fig. 2 is the impact of $c_{1}$ relative to $c_{0}$ on the total flux. One can see that if the velocitydependent component $c_{1}$ provides the dominant contribution to the photon flux, it must be significantly larger (by a few orders of magnitude) than $c_{0}$ need be in order to dominate. This is due to the factor $(v / c)^{2} \sim\left(r_{\mathrm{Sch}} / 2 r\right)$, which is small away from the central black hole. At the end of the day, if there is a velocity-independent contribution to the annihilation cross section, it is likely providing the dominant contribution to the flux. However, it is conceivable that $c_{0}=0$, in which case a very large value of $c_{1}$ could lead to a signal from DM annihilation in the spike, when otherwise no indirect detection signal would be expected [14]. In light of the fact that $c_{0}$ accounts for the lion's share of the photon flux from DM annihilation unless $c_{1} \gg c_{0}$, we will hereafter focus on a benchmark case of DM with an approximately-thermal, velocity-independent annihilation cross section.

Let us pause to briefly discuss the role of $\gamma_{c}$ in predicting the flux of gamma-rays from DM annihilation. When $\gamma_{c}=1.5$, i.e., when a depleted spike would be tiny (see
Fig. 1), the flux of DM annihilation photons from the Sgr A* point source would be three to five orders of magnitude larger than what one would expect from a standard NFW profile ( $\gamma_{c}=1.0$, no spike) due to the cusp itself. For smaller values of $\gamma_{c}$, the flux may include a nonnegligible contribution from the spike, though, even still, the cusp plays an important role. Furthermore, it is only for $c_{1} \gg c_{0}$, that the annihilation rate in the very central region of the spike becomes significantly enhanced by the high velocities of the DM particles such that the velocitydependent contribution to the annihilation cross section is important (see also [13]).

Up to this point, we have focused on $m_{\chi}=100 \mathrm{GeV}$. In Fig. 3, we show the ratio of the flux from DM annihilation relative to $\Phi_{\text {Fermi }}$ as a function of the DM mass for depleted spikes (left) and idealized spikes (right), assuming a benchmark annihilation cross section and $N=1$. In each panel, results are presented for a range of values of $\gamma_{c}$. The horizontal dashed line in each panel shows the point source observational sensitivity $\Phi=\Phi_{\text {Fermi }}$, while the shaded region (the upper limit of which is marked by a vertical contour) shows the mass range excluded by Fermi searches for DM in dwarf galaxies, assuming the final state is either $\tau^{+} \tau^{-}(N=2.5)$ or $b \bar{b}(N=13.5)$ [39]. Note that for this benchmark cross section the sensitivity to both final states is very similar for searches for annihilation in dwarf galaxies since they use spectral information and consider photons with energies as low as $500 \mathrm{MeV}$. For our analysis, on the other hand, the integrated flux scales approximately as $N$. The Fermi Collaboration has also placed constraints on the properties of DM based on the extended emission 

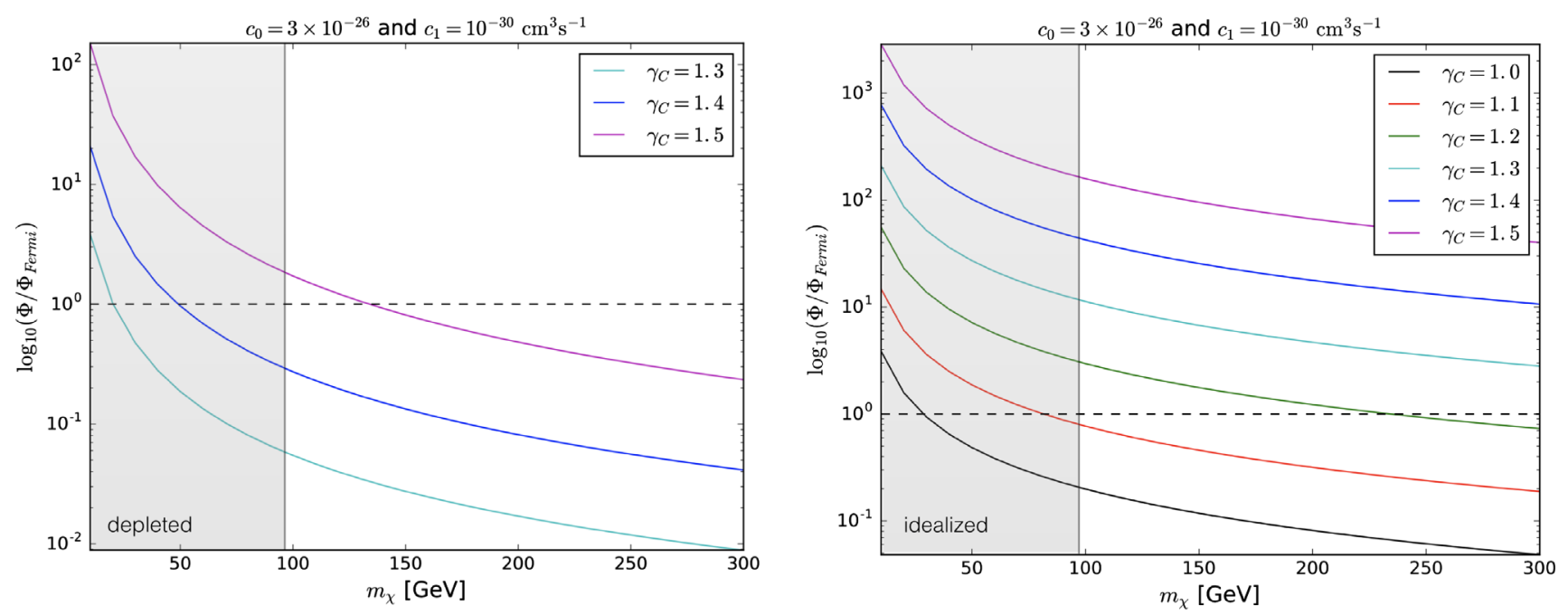

FIG. 3. Observational reach as a function of mass for a depleted spike (left) and an idealized spike (right): In each panel we present the total integrated flux of photons, $\Phi$, coming from the source 3FGL J1745.6-2859c (Sgr A*) in the energy range [1100] GeV, in units of $\Phi_{\text {Fermi }}=2.18 \times 10^{-8}$ photons $/ \mathrm{cm}^{2} \mathrm{~s}$, assuming an integrated photon count $N=1$ for thermal, primarily $s$-wave annihilating dark matter. We assume adiabatic black hole growth, such that the spike profile is given by Eq. (18). In the depleted case (left), the spike radius is given by Eq. (13) with $\tau=10$. The horizontal dashed line shows the observational limit $\Phi=\Phi_{\text {Fermi }}$. The vertical grey line at $m_{\chi}=96 \mathrm{GeV}$ is the upper limit on the dark matter mass from Fermi observations of dwarf galaxies, assuming dark matter annihilates to $\tau^{+} \tau^{-}(N=2.5)[40]$.

from the Galactic center region, indicating that for $c_{0}=3 \times 10^{-26} \mathrm{~cm}^{3} \mathrm{~s}^{-1}, m_{\chi} \gtrsim 30 \mathrm{GeV}$ for annihilation to $b \bar{b}$ and $m_{\chi} \gtrsim 20 \mathrm{GeV}$ for annihilation to $\tau^{+} \tau^{-}$[40]. For the cross section under consideration, these constraints from the Galactic center region are weaker than those from dwarf galaxies, and are therefore not plotted in Fig. 3.

We see that for any DM profile, larger $\gamma_{c}$ leads to an increased integrated flux and higher mass reach. In general, larger values of $\gamma_{c}$ lead to slightly steeper spike profiles inside the spike radius $r_{\mathrm{sp}}$, however the dominant effect comes from $r>r_{\mathrm{sp}}$ where $\gamma_{c}$ determines the flux. For a given/determined $\gamma_{c}$, the sensitivity falls off as $1 / m_{\chi}^{2}$ due to the decreasing number density of DM particles.

In the right panel of Fig. 3, we turn our attention to the possibility that the spike has not depleted as described by Eq. (13), but rather remains intact [41]. Here we see the $1 / m_{\chi}^{2}$ dependence again, but the most striking feature is clearly the much larger mass reach in this case relative to the depleted case in the left panel. If the spike has suffered no depletion, then even very large DM masses are incompatible with large $\gamma_{c}$. Another way of reading this is that if the DM spike at our Galactic center has not suffered much depletion, then the absence of a DM signal gives us an upper limit on $\gamma_{c}$. The effect of depletion, for $\tau=10$, is to decrease the flux from DM annihilation by a factor of $\sim 200$, for any final state or DM mass. Thus, conclusions regarding the viability of a DM model that are sensitive to the presence of a DM spike should be interpreted with extreme caution, in light of the uncertainty as to the exact form of a spike in the DM profile, if such a spike is present at all.
In general, if $\gamma_{c}$ is small, then constraints on DM annihilation from dwarf galaxies are stronger than those due to the overproduction of photons from the point source Sgr A*. This is especially true for depleted spikes (left panel), however if the profile features a spike more similar to the idealized case (right panel), then the constraints from the point source method explored here are comparable to or better than those from dwarf galaxies for $\gamma_{c}=1.0$ and annihilation to a final state with large $N$, such as $b \bar{b}$, or for $\gamma_{c}$ even slightly larger than 1.0 (depending on $N$ ).

Finally, we briefly comment on idealized spikes for which $\gamma_{\text {sp }}$ does not arise solely from adiabatic growth of the black hole. One might expect a smaller value of $\gamma_{\mathrm{sp}}$ than that due to Eq. (18) for a variety of reasons (e.g., those discussed in Sec. II B). If the spike exponent is smaller than the adiabatic expectation, then the sensitivity can be substantially reduced. For example, the flux from an idealized spike with $\gamma_{\mathrm{sp}}=1.8$ (as in [13]) is just a factor of a few larger than the flux from a depleted spike with the same $\gamma_{c}$. In the future, these somewhat degenerate cases may be resolved by carefully studying the extended spatial morphology (rather than just the point source flux) of a gamma-ray signal of DM annihilation.

\section{RESULTS: CONSTRAINTS ON SIMPLIFIED MODELS}

In this section, we present a particular example that demonstrates the impact of the dark matter profile, including a spike, on conclusions regarding the particle physics of DM interactions. Specifically, we describe the constraints 
that are obtained on simplified models of DM with $t$ channel mediators. There is a vast amount of literature on these models, and we refer the reader to [42] and references therein. As above, we take the DM mass to be $100 \mathrm{GeV}$, and consider only $b \bar{b}$ final states. We first describe this class of simplified models and give an overview of the calculation of the annihilation cross section, then we provide a discussion of the results.

\section{A. Simplified model with $\boldsymbol{t}$-channel mediators}

The simplified model we consider includes a Majorana DM candidate $\chi$ that couples to both left- and right-handed SM fermions $f_{L, R}$. The mediator sector consists of a pair of scalars denoted by their mass eigenstates $\tilde{f}_{1,2}$ and a mixing angle $\alpha$ between the scalar mass and chiral eigenstates $\tilde{f}_{L, R}$ given by $[11,12]$

$$
\left(\begin{array}{c}
\tilde{f}_{1} \\
\tilde{f}_{2}
\end{array}\right)=\left(\begin{array}{cc}
\cos \alpha & -\sin \alpha \\
\sin \alpha & \cos \alpha
\end{array}\right)\left(\begin{array}{c}
\tilde{f}_{L} \\
\tilde{f}_{R}
\end{array}\right)
$$

The standard case of a mediator sector coupling to righthanded SM fermions corresponds to the choice $\alpha=\pi / 2$. The interaction Lagrangian is given by

$$
\mathcal{L}_{\text {int }}=\lambda_{L} \tilde{f}_{L}^{*} \bar{\chi} P_{L} f+\lambda_{R} \tilde{f}_{R}^{*} \bar{\chi} P_{R} f+\text { c.c. },
$$

where the Yukawa couplings $\lambda_{L, R}$ may in general contain a $C P$-violating phase, $\lambda_{L} \equiv\left|\lambda_{L}\right| e^{i \varphi / 2}$ and $\lambda_{R} \equiv\left|\lambda_{R}\right| e^{-i \varphi / 2}$. There are thus the following free parameters in this class of simplified models [43]:

(i) the four masses, $m_{\chi}, m_{\tilde{f}_{1}}, m_{\tilde{f}_{2}}$, and $m_{f}$.

(ii) the Yukawas $\left|\lambda_{L, R}\right|$, the scalar mixing angle $\alpha$, and the $C P$-violation phase $\varphi$ (here we take $\varphi=0$ ).

In fact, this simplified model represents a slice of the parameter space of the minimal supersymmetric standard model (MSSM), in which bino DM couples to one generation of light sfermions. In the case of the MSSM, the Yukawa couplings are given by

$$
\begin{aligned}
& \left|\lambda_{L}\right|=\sqrt{2} g\left|Y_{L}\right| \\
& \left|\lambda_{R}\right|=\sqrt{2} g\left|Y_{R}\right|,
\end{aligned}
$$

where $g$ is the electroweak coupling constant and the hypercharges are $\left|Y_{L}\right|=1 / 2$ and $\left|Y_{R}\right|=1$ for leptons and $\left|Y_{L}\right|=1 / 3$ and $\left|Y_{R}\right|=2 / 3$ for quarks.

The relevant diagrams for DM annihilation in this model are given in Fig. 4. Parametrizing the annihilation cross section in the standard way, the velocity-independent $s$-wave contribution $c_{0}$, in the limit $m_{f} / m_{\tilde{f}_{i}} \rightarrow 0$, has a simple periodic dependence on the mixing angle $\alpha$,

$$
c_{0} \propto \cos ^{2} \alpha \sin ^{2} \alpha
$$

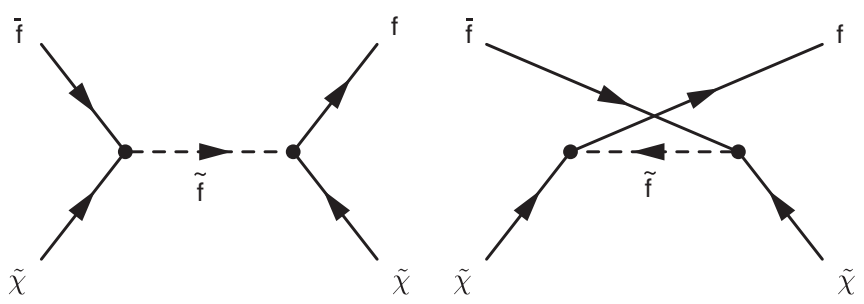

FIG. 4. Feynman diagrams for DM annihilation in the $t$-channel.

with the $v^{2}$-suppressed contribution $c_{1}$ being more complicated (see [43] for details). Here, we use the full expressions for $c_{0}$ and $c_{1}$, including $m_{f}$-suppressed terms.

\section{B. Constraints on simplified models with $t$-channel mediators}

We now discuss the constraints in the context of the simplified model introduced above. We adapt Fig. 2, which gives contours of the flux $\Phi$ in units of the current observational limit $\Phi_{\text {Fermi }}$ in the $\left(c_{0}, c_{1}\right)$ plane, and overlay the cross sections one would obtain in our simplified model.

As an example, we fix the DM and mediator masses and scan over the mixing angle $\alpha$. In Fig. 5, we consider two representative cases: the case of a depleted spike with $\gamma_{c}=$ 1.3 (left), and the case of an idealized spike with $\gamma_{c}=1.0$ (right). The cyan dots show a scan over the mixing angle $\alpha$ defined by Eq. (23), holding the Yukawa couplings fixed at their supersymmetric values given in Eq. (25), and for fixed DM and mediator masses. The scan is performed in the range $\alpha=0$ to $\pi / 2$, which traces out a boomerang in the plane. One could interpret our benchmark model as a supersymmetric realization consisting of bino DM with mass $m_{\chi}=100 \mathrm{GeV}$, a light bottom squark with mass $m_{\tilde{b}}=105 \mathrm{GeV}$, and all other superpartners heavy. The solid black contours represent the observational sensitivity $\Phi / \Phi_{\text {Fermi }}=1,2$.

For the depleted case with $\gamma_{c}=1.3$ considered in the left panel of Fig. 5, we can see that current observational constraints just barely begin to constrain the generic DM parameter space and do not begin to constrain this particular simplified model [44]. If the spike is depleted, then $\gamma_{c} \lesssim 1.3$ is consistent with observation for all values of $\alpha$ for the benchmark masses displayed. Indeed, the constraints are much stronger for the case of an idealized spike, shown in the right panel of Fig. 5. We can see that even for $\gamma_{c}=1.0$, for an idealized spike the current observational limits constrain a large part of the parameter space.

The resulting constraints on $\alpha$ are displayed in Fig. 6 (for the idealized spike in the right panel of Fig. 5). The magenta and cyan curves show the dependence of $c_{0}$ and $c_{1}$ on $\alpha$. For $\alpha \approx 0, \pi / 2$, the annihilation cross section drops precipitously since the contribution from $c_{0}$ suffers from chiral suppression and the contribution from $c_{1}$ is velocity-suppressed. These are the regions where the scans 



FIG. 5. Depleted/Idealized spike, Simplified model—scan over mixing angle $\alpha$ : In the left panel we present the constraints under the assumption of a depleted spike with $\gamma_{c}=1.3$, and in the right panel we assume an idealized spike with $\gamma_{c}=1.0$. In each case, the cyan points correspond to a scan over the mediator mixing angle $\alpha$. The Yukawa couplings are held fixed at their supersymmetric values, given by Eq. (25). The DM mass is $100 \mathrm{GeV}$ and the lightest sbottom mass is $105 \mathrm{GeV}$, with all other superpartners heavy. The solid black line denotes the contour of integrated flux $\Phi=\Phi_{\text {Fermi }}$ coming from Sgr A*, assuming an energy range of 1-100 GeV and $b \bar{b}$ final states for the DM annihilation.

in Fig. 5 are cut off towards the left, where $c_{0}$ becomes small. Conversely, there is a range of values $\alpha \approx 0.08 \pi-0.25 \pi$, where $c_{1}$ becomes small, but $c_{0}$ remains large. These are the regions that are cut off towards the bottom of the scans in Fig. 5, where $c_{1}$ is small.

The horizontal dotted line in Fig. 6 corresponds to $c_{0} \approx 10^{-27} \mathrm{~cm}^{3} \mathrm{~s}^{-1}$, which is where the $\Phi=\Phi_{\text {Fermi }}$ contour for the idealized case in the right panel of Fig. 5 intersects

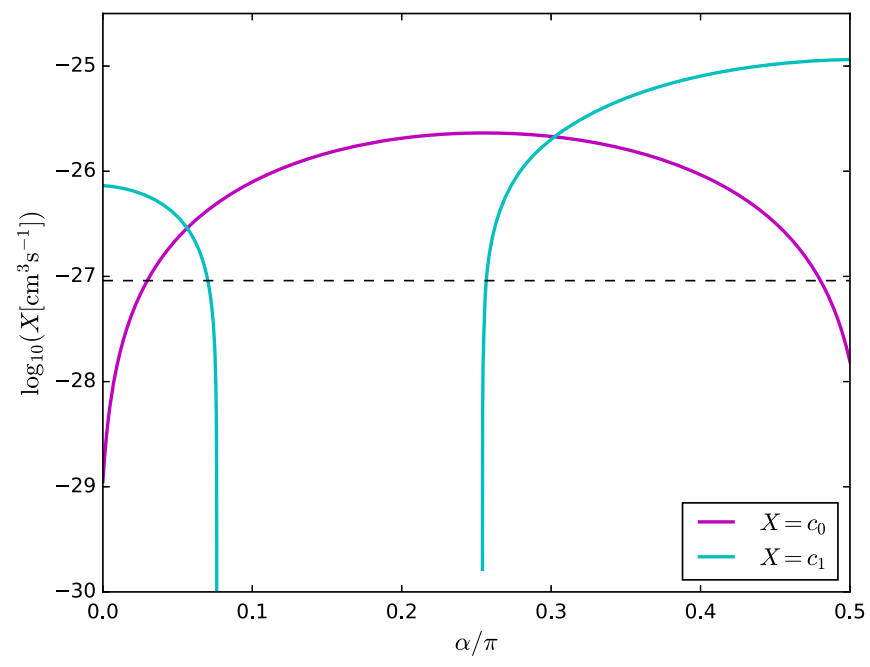

FIG. 6. Dependence of $\mathrm{c}_{0}$ and $\mathrm{c}_{1}$ on $\boldsymbol{\alpha}$ : The purple and blue curves show the dependence of $c_{0}$ and $c_{1}$ on $\alpha$. The horizontal dotted line corresponds to $c_{0} \approx 10^{-27} \mathrm{~cm}^{3} \mathrm{~s}^{-1}$, which is where the $\Phi=\Phi_{\text {Fermi }}$ contour for the idealized case in the right panel of Fig. 5 intersects the $c_{0}$ axis. Values of $c_{0}$ above the dotted line are constrained by the integrated flux of photons coming from Sgr A*. the $c_{0}$ axis. Values of $c_{0}$ larger than this yield an integrated photon flux larger than the observed flux from Sgr A*. Thus, from Fig. 6 , it is clear that either $\alpha \approx 0$ or $\alpha \approx \pi / 2$ if the spike is idealized. Very different conclusions are reached if the spike is depleted.

While this simplified model describes a subset of the MSSM parameter space, it need not be confined to the MSSM. For example, it is possible that the Yukawa couplings, $\lambda_{L, R}$, deviate from their MSSM values. In the absence of a signal, one could then constrain the couplings $\lambda_{L, R}$ for any combination of new particle masses and mixings. If the form of the spike is understood, using the point source flux to constrain the model parameters could be a powerful technique.

\section{CONSTRAINTS ON SPIKE PARAMETERS FROM DM ANNIHILATIONS}

In this section, we invert the approach we have hitherto taken to demonstrate the potential power of gamma-ray observations of a known DM candidate to determine the spike profile (and potentially learn something about the astrophysics that led to it). Although the most recent analysis indicates that the excess of $\mathrm{GeV}$ photons from the Galactic center region observed by Fermi-LAT is most likely not due to DM [40], it is instructive to take this case as an example. We calculate the constraints on the spike parameters in our model under the assumption of a particular DM model designed to explain the excess of $\sim 1-3 \mathrm{GeV}$ gamma-rays from the Galactic center. Specifically, we take as our benchmark point 


$$
\begin{aligned}
m_{\chi} & =49 \mathrm{GeV} \\
c_{0} & =1.76 \times 10^{-26} \mathrm{~cm}^{3} \mathrm{~s}^{-1} \\
c_{1} & =1.0 \times 10^{-30} \mathrm{~cm}^{3} \mathrm{~s}^{-1},
\end{aligned}
$$

and assume $b \bar{b}$ final states, as in [22-24].

Clearly, many choices for the spike parameters and the relationships among them exist, and considering different combinations would lead to different kinds of constraints on the parameter space. As a representative case, in Fig. 7 we consider a depleted spike and present our results in the $\left(\gamma_{c}, \gamma_{\mathrm{sp}}\right)$ plane. Though we do not explicitly enforce the adiabatic relation for $\gamma_{\mathrm{sp}}$, we plot it as a dashed line in the plane for reference. The solid black contours denote the integrated flux $\Phi / \Phi_{\text {Fermi. }}$. It is clear that for a depleted spike, $\gamma_{c} \gtrsim 1.3$ is incompatible with a DM interpretation of the Galactic center excess for most values of $\gamma_{\mathrm{sp}}$. This is even true for very steep spikes with large $\gamma_{\mathrm{sp}}$; as long as $\gamma_{c}$ is not too large, these scenarios are not excluded by the point source flux.

Additionally, while the spike itself would contribute to the photon flux if the spike is idealized, the fact that the contours in Fig. 7 are nearly independent of $\gamma_{\mathrm{sp}}$, i.e., mostly vertical, further demonstrates that for this depleted case it is not actually the spike that is responsible for the bulk of the photons. Instead, the spike is fairly insignificant relative to

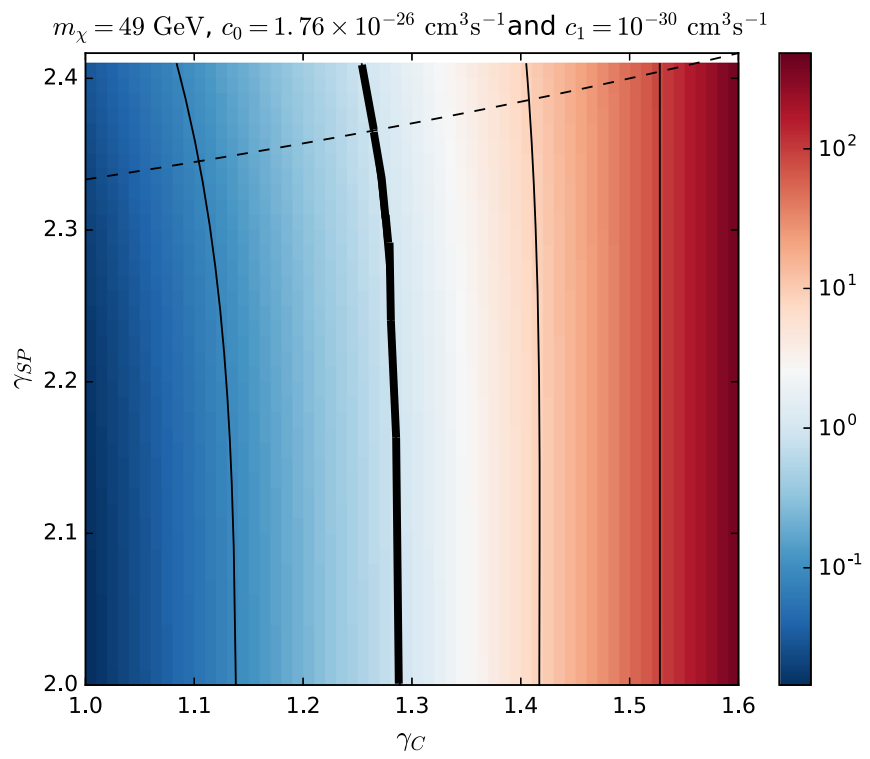

FIG. 7. Constraints on spike parameters, assuming depleted spike and Galactic center excess: We assume a DM mass of $49 \mathrm{GeV}$ and an annihilation cross section parametrized by Eq. (4) with $c_{0}=1.76 \times 10^{-26} \mathrm{~cm}^{3} \mathrm{~s}^{-1}$ and $c_{1}=1.0 \times 10^{-30} \mathrm{~cm}^{3} \mathrm{~s}^{-1}$. The solid black contours denote the integrated flux $\Phi / \Phi_{\text {Fermi }}$ coming from the point source Sgr $\mathrm{A}^{*}$, assuming an energy range of $1-100 \mathrm{GeV}$ and $b \bar{b}$ final states in DM annihilation. The bold contour corresponds to $\Phi=\Phi_{\mathrm{Fermi}}$. The dotted line shows the relation between $\gamma_{\mathrm{sp}}$ and $\gamma_{c}$ given by Eq. (18) for the purely adiabatic case. the smooth component of the halo, characterized by $\gamma_{c}$. Ultimately, with some knowledge of the properties of DM, perhaps an observed, or unobserved, flux may help us learn about the DM profile near the Galactic center, and possibly even the astrophysical mechanisms at play.

\section{CONCLUSIONS}

In this paper, we have explored the impacts of the modeling of the dark matter distribution in our Galaxy, including a potential dark matter spike at the Galactic center, on indirect signals of dark matter annihilation. Taking Fermi-LAT data for the gamma-ray flux from the point source 3FGL J1745.6-2859c (Sgr A*), we calculated the resulting constraints on generic models of DM, allowing for the possibility of a non-negligible velocitydependent contribution to the annihilation cross section. We find that the spike formation history and profile parameters have a profound effect on the extent to which models of DM can be constrained.

(i) For the most conservative choice of parameters (those which yield the smallest dark matter signal, i.e., a depleted spike with radius given by Eq. depletion and $\gamma_{c}=1.0$ ), the flux for a $100 \mathrm{GeV}$ thermal relic is several order of magnitude below current observational limits. We have then considered a series of potential dark matter profiles that give larger contributions to the gamma ray flux from the Galactic center point source, Sgr A*.

(ii) A depleted spike with steeper cusp profile would constrain thermal relics of different masses depending on $\gamma_{c}$, as shown in the left panel of Fig. 3. Note that the constraints in this case are due to the flux from the cusp, as the spike itself is relatively insignificant (see eg. Fig. 7). We see that thermal relics with masses of $\mathcal{O}(10-100) \mathrm{GeV}$ are constrained for $\gamma_{c} \gtrsim 1.3$, with exact constraints depending on the number of photons in the relevant energy range produced in each annihilation, $N$.

(iii) An idealized spike which has not undergone depletion improves the constraints considerably; the mass reach is shown in the right panel of Fig. 3. This assumes that the inner spike profile corresponds to a scenario where the DM spike formed in response to the adiabatic growth of the black hole, i.e., $\gamma_{\mathrm{sp}} \sim 2.3-2.4$. We see that thermal relics with masses of $\mathcal{O}(10-100) \mathrm{GeV}$ (depending on $N$ ) are constrained even for small to moderate values of $\gamma_{c}$ (1.0-1.2).

(iv) Finally, if the spike is depleted and the profile is flatter than one would expect from strictly adiabatic black hole growth, then, in general, the flux of gamma rays from dark matter annihilation is nearly entirely from the cusp rather than the spike. This is demonstrated in Fig. 7, in that the contours of constant flux are vertical up to large values of $\gamma_{\mathrm{sp}}$. 
In Sec. IV, we applied our analysis to a simplified model of fermionic DM with $t$-channel mediators described by Eq. (24). We found that if the spike is depleted, constraints on the DM model are possible only if $\gamma_{c} \gtrsim 1.3$, but if the spike is idealized then large parts of the parameter space are constrained, even for a cusp profile of $\gamma_{c}=1.0$. Constraints on the dark matter model space are very sensitive to depletion effects.

In Sec. V we explored the possibility of constraining the space of astrophysical spike parameters, assuming that we know something about the properties of the DM, taking as an example a proposed DM candidate to explain the excess of $\mathrm{GeV}$ photons from the Galactic center observed by Fermi-LAT. If the spike is depleted, we find that moderate values of $\gamma_{c} \lesssim 1.3$ would be compatible with this particular model of DM, a conclusion which is largely independent of $\gamma_{\mathrm{sp}}$. Furthermore, very large values of $\gamma_{c}$ could certainly be excluded based on overproduction of point source photons associated with Sgr A*.

Finally, we would like to note that the depletion we assume is for a heating timescale of $10^{9} \mathrm{yr}$, which may be either shorter or longer than is realized in nature. If depletion is less strong, which here might be realized by a longer heating timescale, then the fluxes from any given model would be larger. This means that the power to probe DM models would be greater, or, conversely, the power to use some knowledge about the properties of DM to constrain $\gamma_{\mathrm{sp}}$ and $\gamma_{c}$ would be greater than in the depleted scenarios presented here.

\section{ACKNOWLEDGMENTS}

We would like to thank Mustafa Amin for collaboration in the early stages of this work. P. S. is supported in part by NSF Grants No. PHY-1417367 and No. PHY-1720282.
[1] R. Genzel et al., The stellar cusp around the supermassive black hole in the Galactic Center, Astrophys. J. 594, 812 (2003).

[2] R. Schodel, T. Ott, R. Genzel, A. Eckart, N. Mouawad, and T. Alexander, Stellar dynamics in the central arcsecond of our galaxy, Astrophys. J. 596, 1015 (2003).

[3] P. Gondolo and J. Silk, Dark Matter Annihilation at the Galactic Center, Phys. Rev. Lett. 83, 1719 (1999).

[4] P. Ullio, H. Zhao, and M. Kamionkowski, A dark-matter spike at the Galactic center?, Phys. Rev. D 64, 043504 (2001).

[5] D. Merritt, Evolution of the Dark Matter Distribution at the Galactic Center, Phys. Rev. Lett. 92, 201304 (2004).

[6] G. Bertone and D. Merritt, Time-dependent models for dark matter at the Galactic center, Phys. Rev. D 72, 103502 (2005).

[7] O. Y. Gnedin and J. R. Primack, Dark Matter Profile in the Galactic Center, Phys. Rev. Lett. 93, 061302 (2004).

[8] E. J. Ahn, G. Bertone, and D. Merritt, Impact of astrophysical processes on the gamma-ray background from dark matter annihilations, Phys. Rev. D 76, 023517 (2007).

[9] J. Shelton, S. L. Shapiro, and B. D. Fields, Black Hole Window into $p$-Wave Dark Matter Annihilation, Phys. Rev. Lett. 115, 231302 (2015).

[10] M. A. Amin and T. Wizansky, Relativistic dark matter at the Galactic center, Phys. Rev. D 77, 123510 (2008).

[11] P. Sandick, K. Sinha, and F. Teng, Simplified dark matter models with charged mediators: Prospects for direct detection, J. High Energy Phys. 10 (2016) 018.

[12] J. Kumar, P. Sandick, F. Teng, and T. Yamamoto, Gammaray signals from dark matter annihilation via charged mediators, Phys. Rev. D 94, 015022 (2016).

[13] B. D. Fields, S. L. Shapiro, and J. Shelton, Galactic Center Gamma-Ray Excess from Dark Matter Annihilation:
Is There A Black Hole Spike?, Phys. Rev. Lett. 113, 151302 (2014).

[14] S. L. Shapiro and J. Shelton, Weak annihilation cusp inside the dark matter spike about a black hole, Phys. Rev. D 93, 123510 (2016).

[15] P. Sandick and S. Watson, Constraints on a non-thermal history from Galactic dark matter spikes, Phys. Rev. D 84, 023507 (2011).

[16] P. Sandick, J. Diemand, K. Freese, and D. Spolyar, Signatures of dark star remnants in the Galactic halo, Proc. Sci., IDM2010 (2011) 086 [arXiv:1012.0068].

[17] P. Sandick, J. Diemand, K. Freese, and D. Spolyar, Black holes in our galactic halo: Compatibility with FGST and PAMELA data and constraints on the first stars, J. Cosmol. Astropart. Phys. 01 (2011) 018.

[18] D. Schoonenberg, J. Gaskins, G. Bertone, and J. Diemand, Dark matter subhalos and unidentified sources in the Fermi 3FGL source catalog, J. Cosmol. Astropart. Phys. 05 (2016) 028 .

[19] M. Wanders, G. Bertone, M. Volonteri, and C. Weniger, No WIMP mini-spikes in dwarf spheroidal galaxies, J. Cosmol. Astropart. Phys. 04 (2015) 004.

[20] A. X. Gonzalez-Morales, S. Profumo, and F. S. Queiroz, Effect of black holes in local dwarf spheroidal galaxies on gamma-ray constraints on dark matter annihilation, Phys. Rev. D 90, 103508 (2014).

[21] T. Lacroix, C. Bhm, and J. Silk, Ruling out thermal dark matter with a black hole induced spiky profile in the M87 galaxy, Phys. Rev. D 92, 043510 (2015).

[22] T. Daylan, D. P. Finkbeiner, D. Hooper, T. Linden, S. K. N. Portillo, N. L. Rodd, and T. R. Slatyer, The characterization of the gamma-ray signal from the central Milky Way: A case for annihilating dark matter, Phys. Dark Universe 12, 1 (2016); F. Calore, I. Cholis, and C. Weniger, Background 
model systematics for the Fermi GeV excess, J. Cosmol. Astropart. Phys. 03 (2015) 038; D. Hooper, The density of dark matter in the galactic bulge and implications for indirect detection, Phys. Dark Universe 15, 53 (2017).

[23] C. Karwin, S. Murgia, T. M. P. Tait, T. A. Porter, and P. Tanedo, Dark matter interpretation of the Fermi-LAT observation toward the Galactic center, Phys. Rev. D 95, 103005 (2017).

[24] M. Ajello et al. (Fermi-LAT Collaboration), Fermi-LAT observations of high-energy $\gamma$-ray emission toward the Galactic center, Astrophys. J. 819, 44 (2016).

[25] M. Srednicki, R. Watkins, and K. A. Olive, Calculations of relic densities in the early universe, Nucl. Phys. B310, 693 (1988).

[26] L. Ferrarese and H. Ford, Supermassive black holes in galactic nuclei: Past, present and future research, Space Sci. Rev. 116, 523 (2005).

[27] G. Bertone and D. Merritt, Dark matter dynamics and indirect detection, Mod. Phys. Lett. A 20, 1021 (2005).

[28] D. Merritt, Single and binary black holes and their influence on nuclear structure, arXiv:astro-ph/0301257.

[29] J. F. Navarro, C. S. Frenk, and S. D. M. White, The structure of cold dark matter halos, Astrophys. J. 462, 563 (1996).

[30] J. Diemand, M. Kuhlen, P. Madau, M. Zemp, B. Moore,

D. Potter, and J. Stadel, Clumps and streams in the local dark matter distribution, Nature (London) 454, 735 (2008).

[31] J. F. Navarro, A. Ludlow, V. Springel, J. Wang, M. Vogelsberger, S. D. M. White, A. Jenkins, C. S. Frenk, and A. Helmi, The diversity and similarity of cold dark matter halos, Mon. Not. R. Astron. Soc. 402, 21 (2010).

[32] O. Y. Gnedin, A. V. Kravtsov, A. A. Klypin, and D. Nagai, Response of dark matter halos to condensation of baryons: Cosmological simulations and improved adiabatic contraction model, Astrophys. J. 616, 16 (2004).

[33] M. Gustafsson, M. Fairbairn, and J. Sommer-Larsen, Baryonic pinching of galactic dark matter haloes, Phys. Rev. D 74, 123522 (2006).

[34] M. Pato, F. Iocco, and G. Bertone, Dynamical constraints on the dark matter distribution in the Milky Way, J. Cosmol. Astropart. Phys. 12 (2015) 001.
[35] E. Vasiliev, Dark matter annihilation near a black hole: Plateau vs. weak cusp, Phys. Rev. D 76, 103532 (2007).

[36] T. Sjostrand, S. Mrenna, and P.Z. Skands, PYTHIA 6.4 physics and manual, J. High Energy Phys. 05 (2006) 026.

[37] K. N. Abazajian, N. Canac, S. Horiuchi, and M. Kaplinghat, Astrophysical and dark matter interpretations of extended gamma-ray emission from the Galactic center, Phys. Rev. D 90, 023526 (2014).

[38] Choosing a small angular acceptance rather than using the full PSF may underestimate the flux from the spike by a factor of a few, depending on the final state. Here, we generally prefer agnosticism regarding the annihilations themselves, and are interested in broad trends in detectability. We will see that the exponents $\gamma_{c}$ and $\gamma_{\mathrm{sp}}$ can cause variations in the flux by several orders of magnitude.

[39] M. Ackermann et al. (Fermi-LAT Collaboration), Searching for Dark Matter Annihilation from Milky Way Dwarf Spheroidal Galaxies with Six Years of Fermi Large Area Telescope Data, Phys. Rev. Lett. 115, 231301 (2015).

[40] M. Ackermann et al. (Fermi-LAT Collaboration), The Fermi Galactic center $\mathrm{GeV}$ excess and implications for dark matter, Astrophys. J. 840, 43 (2017).

[41] The spike may also be described by a spike exponent different from the adiabatic expectation: a value of $\gamma_{\mathrm{sp}}$ different from Eq. (18) could arise at formation or through a type of depletion different from that considered here, as briefly described in Sec. II B. We refer to both of these cases as idealized in the sense that $r_{\mathrm{sp}}$ is unchanged from its initial value.

[42] M. Garny, A. Ibarra, and S. Vogl, Signatures of Majorana dark matter with t-channel mediators, Int. J. Mod. Phys. D 24, 1530019 (2015).

[43] K. Fukushima, C. Kelso, J. Kumar, P. Sandick, and T. Yamamoto, MSSM dark matter and a light slepton sector: The incredible bulk, Phys. Rev. D 90, 095007 (2014); K. Fukushima and J. Kumar, Dipole moment bounds on dark matter annihilation, Phys. Rev. D 88, 056017 (2013).

[44] For $\gamma_{c}=1.0$, as one would expect, the fluxes are far below $\Phi_{\text {Fermi }}$, while for $\gamma_{c}=1.5$ a large fraction of the parameter space is constrained. 